\title{
Dynamics of a diffusive viral model with Beddington-DeAngelis incidence rate and CTL immune response
}

\section{Kejun Zhuang}

School of Statistics and Applied Mathematics, Anhui University of Finance and Economics, Bengbu 233030, China.

\author{
Communicated by Y.-Z. Chen
}

\begin{abstract}
In this paper, a four-dimensional system of viral model with cytotoxic lymphocyte (CTL) immune response is investigated. This model is a reaction-diffusion system with Beddington-DeAngelis incidence rate and free diffusion in a bounded domain. With the help of comparison principle and Lyapunov function method, the well-posedness of solutions and sufficient conditions for global stability of nonnegative equilibria are established. It can be found that free diffusion has no influence on the global stability of the system with homogeneous Neumann boundary conditions. (c)2017 All rights reserved.
\end{abstract}

Keywords: Viral model, global stability, Beddington-DeAngelis incidence rate, CTL immune response. 2010 MSC: 35B35, 35K57, 92D25.

\section{Introduction}

Viral infections can cause illnesses as minor as the common cold and as severe as hepatitis or even acquired immune deficiency syndrome (AIDS). To better understand the infection process, mathematical models have played an important part and attracted many researchers' attention. In fact, some classic mathematical models have been used to model the dynamics of viral infections such as human immunodeficiency virus (HIV) and hepatitis B virus (HBV) infection, see $[6,8,11-14,20,22]$ and the references therein.

The basic model describing the interaction between the susceptible host cells (hepatocytes, $x$ ), infection host cells $(y)$, and free virus particles $(v)$, is formulated by the following differential equations [11-13]:

$$
\left\{\begin{array}{l}
\frac{d x}{d t}=\lambda-d x-\beta x v, \\
\frac{d y}{d t}=\beta x v-p y, \\
\frac{d v}{d t}=k y-u v,
\end{array}\right.
$$

where hepatocytes are produced at a rate $\lambda$, die at a rate $d x$, and become infected at a rate $\beta x v$; infected hepatocytes are produced at a rate $\beta \times v$ and die at a rate py; free viruses are produced from infected

Email address: zhkj1230163.com (Kejun Zhuang)

doi:10.22436/jnsa.010.11.13 
cells at a rate $k y$ and are removed at a rate $u v$. It is assumed that parameters $\lambda, d, \beta, p, k, u$ are all positive constants. Moreover, the rate of infection in (1.1) is supposed to be bilinear. However, the actual incidence rate probably is not linear and it is more reasonable to assume that the infection rate is nonlinear $[1,4,17,26]$. Huang et al. [7] considered the Beddington-DeAngelis functional response and modified the model as follows

$$
\left\{\begin{array}{l}
\frac{d x}{d t}=\lambda-d x-\frac{\beta x v}{1+a x+b v} \\
\frac{d y}{d t}=\frac{\beta x v}{1+a x+b v}-p y, \\
\frac{d v}{d t}=k y-u v
\end{array}\right.
$$

When $a>0$ and $b=0$, the specific functional response is reduced to Holling type II function response [9]. And when $a=0, b>0$, it is simplified to be a saturation response [15]. Moreover, when $a=b=0$, the specific functional response expresses the mass action process (or Holling type I functional response).

If one is infected by some virus, the immune system may act and protect the body from possibly harmful substances by recognizing and responding to antigens. And in most virus infections, cytotoxic lymphocyte cells (CTLs) and antibody cells play a critical part in antiviral defense [11]. Therefore, Wang et al. [18] proposed the following system with nonlinear incidence rate describing immune response against infected cells:

$$
\left\{\begin{array}{l}
\frac{\mathrm{d} x}{\mathrm{~d} t}=\lambda-\mathrm{d} x-\frac{\beta x v}{1+\mathrm{m} x+n v} \\
\frac{\mathrm{d} y}{\mathrm{~d} t}=\frac{\beta x v}{1+\mathrm{m} x+n v}-\mathrm{a} y-\mathrm{pyz} \\
\frac{\mathrm{d} v}{\mathrm{~d} t}=\mathrm{k} y-\mathrm{uv} \\
\frac{\mathrm{d} z}{\mathrm{dt}}=\mathrm{cyz}-\mathrm{bz}
\end{array}\right.
$$

where $z(t)$ denotes the concentration of CTLs.

Furthermore, the influences of spatial structures on virus dynamics can not be ignored [19]. But so far, there have been very few results about the influences of spatial structures on virus dynamics with immune response and Beddington-DeAngelis incidence rate. Motivated by these factors, we introduce the random mobility of viruses into system (1.3) and assume that the motion of virus follows the Fickian diffusion [2]. From above, we can obtain the following reaction-diffusion model:

$$
\left\{\begin{array}{l}
\frac{\partial u(x, t)}{\partial t}=\lambda-d u(x, t)-\frac{k u(x, t) v(x, t)}{1+\alpha u(x, t)+\beta v(x, t)}, \\
\frac{\partial w(x, t)}{\partial t}=\frac{k u(x, t) v(x, t)}{1+\alpha u(x, t)+\beta v(x, t)}-a w(x, t)-p w(x, t) z(x, t), \\
\frac{\partial v(x, t)}{\partial t}=D \Delta v(x, t)+q w(x, t)-m v(x, t), \\
\frac{\partial z(x, t)}{\partial t}=c w(x, t) z(x, t)-b z(x, t),
\end{array}\right.
$$

where $u(x, t), w(x, t), v(x, t)$, and $z(x, t)$ represent the densities of uninfected cells, infected cells, free virus, and CTLs at location $x \in \Omega \subseteq \mathbb{R}^{n}$ and time $t$, respectively. All the parameters are positive constants. $\Delta v=\sum_{i=1}^{n} \frac{\partial^{2} v}{\partial x_{i}^{2}}(n=1,2$ or 3$)$ is the Laplacian operator, $D$ is the diffusion coefficient.

For system (1.4), we consider the initial value conditions

$$
u(x, 0)=\phi_{1}(x), \quad w(x, 0)=\phi_{2}(x), \quad v(x, 0)=\phi_{3}(x), \quad z(x, 0)=\phi_{4}(x), \quad \forall x \in \bar{\Omega}
$$

and the Neumann boundary conditions

$$
\frac{\partial u}{\partial v}=\frac{\partial w}{\partial v}=\frac{\partial v}{\partial v}=\frac{\partial z}{\partial v}=0, \quad t>0, \quad x \in \partial \Omega,
$$

where $\phi_{i}(x)(i=1,2,3,4)$ are nonnegative and Hölder continuous in $\bar{\Omega}$, and $\partial / \partial v$ denotes the outward normal derivative on $\partial \Omega$. The Neumann boundary conditions mean that the bound is fixed and virus population does not emigrate or immigrate across the boundary $\partial \Omega$.

Since spatial diffusion does not change the number and location of constant equilibria, we can establish the existence of nonnegative equilibria of (1.4), which is consistent with the results in [18]. 
Lemma 1.1. The following conclusions about the nonnegative equilibria of model (1.4) hold:

(i) the model always has the disease-free equilibrium $\mathrm{E}_{0}=(\lambda / \mathrm{d}, 0,0,0)$;

(ii) if the basic reproductive number $R_{0}>1$, then there exists an immune-free equilibrium $E_{1}=\left(u_{1}, w_{1}, v_{1}, 0\right)$, where

$$
\begin{aligned}
\mathrm{R}_{0} & =\frac{\lambda k q}{\mathrm{~m}(\mathrm{ad}+\mathrm{a} \alpha \lambda)}, & \mathrm{u}_{1} & =\frac{a m+\beta \lambda q}{\mathrm{kq}+\beta d q-a m \alpha}, \\
w_{1} & =\frac{\lambda k q\left(\mathrm{R}_{0}-1\right)}{a R_{0}(k q+\beta d q-a m \alpha)}, & v_{1} & =\frac{\lambda k q^{2}\left(\mathrm{R}_{0}-1\right)}{a m R_{0}(k q+\beta d q-a m \alpha)} ;
\end{aligned}
$$

(iii) if the immune response reproduction number $\mathrm{R}^{0}>1$, then there exists an interior equilibrium $\mathrm{E}^{*}=\left(\mathrm{u}^{*}, w^{*}, v^{*}\right.$, $\left.z^{*}\right)$, where

$$
\begin{aligned}
& u^{*}=\frac{-\left(d+\beta d v^{*}+k v^{*}-\alpha \lambda\right)+\sqrt{\left(d+d \beta v^{*}+k v^{*}-\alpha \lambda\right)^{2}+4 d \alpha \lambda\left(1+\beta v^{*}\right)}}{2 d \alpha}, \quad w^{*}=\frac{b}{c}, \\
& v^{*}=\frac{q}{m} w^{*}, \quad z^{*}=\frac{\lambda-d u^{*}-a w^{*}}{p w^{*}}, \quad R^{0}=\frac{c w_{1}}{b}=\frac{\lambda k q c+a^{2} b m \alpha}{a(c d m+b d q \beta+b k q+c m \alpha \lambda)} .
\end{aligned}
$$

For the special cases of model (1.4), some important results have been obtained. For example, Huang et al. [7] analyzed the global properties of system (1.2) in the absence of CTL immune response and spatial diffusion. Wang et al. [18] studied the global stability of nonnegative equilibria in system (1.3) without considering the spatial diffusion. In [19], Wang et al. ignored the immune response and established the existence of traveling waves when $\alpha=\beta=0$. However, whether the movement of virus has influences on the dynamics of viral model is still an interesting and significant theme. As a result, the main purpose of the present paper is to explore the dynamics of the diffusive viral model (1.4). The similar research can be found in $[23,25]$.

The rest of paper is organized as follows. In Section 2, the well-posedness of solutions and dissipativeness are discussed. In Section 3, the sufficient conditions for global stability of three nonnegative equilibria are obtained. Finally, a brief discussion is given in Section 4.

\section{The well-posedness and dissipativeness}

In this section, because of the biological background, we mainly establish the existence, uniqueness, positivity, and boundedness of solutions for system (1.4) with initial value conditions (1.5) and boundary conditions (1.6). We will also establish the dissipativeness.

Theorem 2.1. For problem (1.4)-(1.6), there exists a unique solution defined on $[0,+\infty)$ and this solution is nonnegative and uniformly bounded for all $\mathrm{t} \geqslant 0$.

Proof. By standard existence theory in [21], it is not hard to deduce the local existence of the unique solution $(u(x, t), w(x, t), v(x, t), z(x, t))$ of $(1.4)$ for $x \in \bar{\Omega}$ and $t \in[0, T)$, where $T$ is the maximal existence time for solution.

In addition, we have $u(x, t) \geqslant 0, w(x, t) \geqslant 0, v(x, t) \geqslant 0$, and $z(x, t) \geqslant 0$ because $(0,0,0,0)$ is a lower solution of system (1.4).

Next, we prove the boundedness of solutions. From the nonnegativity of solutions and the first equation of (1.4), we have

$$
\frac{\partial u(x, t)}{\partial t} \leqslant \lambda-d u(x, t) \quad \text { for } \quad(x, t) \in \Omega \times[0, T) .
$$

The comparison principle shows that

$$
u(x, t) \leqslant \max \left\{\frac{\lambda}{d}, \sup _{x \in \bar{\Omega}}\left\|\phi_{1}(x)\right\|\right\}=M_{1} \quad \text { for } t \in[0, T) .
$$


Similarly, we have

$$
w(x, t) \leqslant \max \left\{\frac{k M_{1}}{a \beta} \sup _{x \in \bar{\Omega}}\left\|\phi_{2}(x)\right\|\right\}=M_{2} \quad \text { for } \quad t \in[0, T)
$$

and

$$
v(x, t) \leqslant \max \left\{\frac{q M_{2}}{m}, \sup _{x \in \bar{\Omega}}\left\|\phi_{3}(x)\right\|\right\}=M_{3} \quad \text { for } \quad t \in[0, T) .
$$

From the second and fourth equations of (1.4), we can obtain

$$
\frac{c}{p} \frac{\partial u(x, t)}{\partial t}+\frac{\partial z(x, t)}{\partial t} \leqslant \frac{c k M_{1}}{p \beta}-\frac{a c}{p} w(x, t)-b z(x, t), \quad \text { for } \quad(x, t) \in \Omega \times[0, T),
$$

thus,

$$
z(x, t) \leqslant \frac{c}{p} u(x, t)+z(x, t) \leqslant \max \left\{\frac{c k M_{1}}{p \beta \gamma}, \frac{c}{p} \sup _{x \in \bar{\Omega}}\left\|\phi_{2}(x)\right\|+\sup _{x \in \bar{\Omega}}\left\|\phi_{4}(x)\right\|\right\}=M_{4}
$$

for $t \in[0, T)$, where $\gamma=\min \{a c / p, b\}$.

Note that the upper bound of solution is independent of the maximal existence interval $[0, T)$, we have $\mathrm{T}=+\infty$ due to the standard theory for semilinear parabolic systems [5]. This completes the proof.

Theorem 2.2 (Dissipativeness). The nonnegative solution $(u, w, v, z)$ of system (1.4) satisfies

$$
\begin{array}{ll}
\limsup _{t \rightarrow+\infty} u(x, t) \leqslant \frac{\lambda}{d}, & \limsup _{t \rightarrow+\infty}(x, t) \leqslant \frac{k \lambda}{a d \beta}, \\
\limsup _{t \rightarrow+\infty} v(x, t) \leqslant \frac{k q \lambda}{a d m \beta}, & \limsup _{t \rightarrow+\infty}(x, t) \leqslant \frac{c k \lambda}{d p \beta \gamma} .
\end{array}
$$

Proof. From the first equation of (1.4), we have

$$
\frac{\partial u(x, t)}{\partial t} \leqslant \lambda-d u(x, t) \text { for }(x, t) \in \Omega \times[0,+\infty),
$$

the first result easily follows from the simple comparison principle, and thus there exists $T_{1} \in(0,+\infty)$ such that $u(x, t) \leqslant \lambda / d+\varepsilon_{1}$ in $\Omega \times\left[T_{1},+\infty\right)$ for an arbitrary constant $\varepsilon_{1}>0$.

Then we have

$$
\frac{\partial w(x, t)}{\partial t} \leqslant \frac{k}{\beta}\left(\frac{\lambda}{d}+\varepsilon_{1}\right)-a w(x, t) \text { for }(x, t) \in \Omega \times\left[T_{1},+\infty\right)
$$

and

$$
\limsup _{t \rightarrow+\infty} w(x, t) \leqslant \frac{k}{a \beta}\left(\frac{\lambda}{d}+\varepsilon_{1}\right)
$$

which implies

$$
\limsup _{t \rightarrow+\infty} w(x, t) \leqslant \frac{k \lambda}{\operatorname{ad} \beta}
$$

by the continuity as $\varepsilon_{1} \rightarrow 0$.

Therefore, there exists $T_{2}>T_{1}$ such that $w(x, t) \leqslant \frac{k \lambda}{a d \beta}+\varepsilon_{2}$ in $\Omega \times\left[T_{2},+\infty\right)$ for an arbitrary constant $\varepsilon_{2}>0$.

By the third equation of (1.4), we get

$$
\frac{\partial v(x, t)}{\partial t}-D \Delta v(x, t) \leqslant q\left(\frac{k \lambda}{a d \beta}+\varepsilon_{2}\right)-m v(x, t) \text { for }(x, t) \in \Omega \times\left[T_{1},+\infty\right) .
$$


The comparison principle shows that

$$
\limsup _{t \rightarrow+\infty} v(x, t) \leqslant \frac{q}{m}\left(\frac{k \lambda}{a d \beta}+\varepsilon_{2}\right)
$$

and

$$
\limsup _{t \rightarrow+\infty} v(x, t) \leqslant \frac{k q \lambda}{\operatorname{adm} \beta}
$$

by the arbitrariness of $\varepsilon_{2}$.

Multiplying the second equation by $\frac{c}{p}$ and adding it to the fourth equation in system (1.4), we have

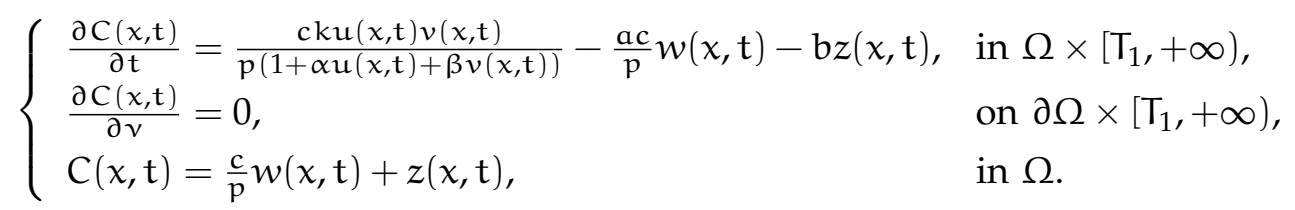

The comparison principle leads to

$$
\limsup _{t \rightarrow+\infty} z(x, t) \leqslant \limsup _{t \rightarrow+\infty} C(x, t) \leqslant \frac{c k}{p \beta \gamma}\left(\frac{\lambda}{d}+\varepsilon_{1}\right)
$$

on $\bar{\Omega}$, and thus $\lim _{t \rightarrow+\infty} \sup z(x, t) \leqslant \frac{c k \lambda}{\operatorname{dp} \beta \gamma}$ by the continuity as $\varepsilon_{1} \rightarrow 0$. The proof is complete.

Remark 2.3. From Theorem 2.2, we know that $A=\left[0, \frac{\lambda}{d}\right] \times\left[0, \frac{k \lambda}{\operatorname{ad} \beta}\right] \times\left[0, \frac{k q \lambda}{\operatorname{adm} \beta}\right] \times\left[0, \frac{c k \lambda}{d p \beta \gamma}\right]$ is a global attractor for all solutions of system (1.4) in the sense that any nonnegative solution lies in $A$ as $t \rightarrow+\infty$ for all $x \in \Omega$.

\section{Global stability}

In this section, we are concerned with the influence of spatial diffusion on global stability of diseasefree equilibrium $E_{0}$, immune-free equilibrium $E_{1}$ and interior equilibrium $E^{*}$, respectively. The methods here are to use comparison techniques and construct suitable Lyapunov function.

\subsection{Global stability of the disease-free equilibrium}

Theorem 3.1. If $\mathrm{R}_{0}<1$, then the disease-free equilibrium $\mathrm{E}_{0}$ of system (1.4) is globally asymptotically stable.

Proof. From the assumption $R_{0}<1$, we can choose sufficiently small $\varepsilon_{1}>0$ such that $\lambda k q+(d k q-$ $\operatorname{adm} \alpha) \varepsilon_{1} \leqslant \operatorname{adm}+a m \alpha \lambda$. For this $\varepsilon_{1}$, according to the results in Theorem 2.2, there exists $\mathrm{T}_{1}>0$ such that $u(x, t) \leqslant \lambda / d+\varepsilon_{1}$ in $\Omega \times\left[T_{1},+\infty\right)$.

Multiplying the third equation by $\frac{a}{q}$ and the fourth equation by $\frac{p}{c}$, respectively, then adding them to the second equation in system (1.4), we have

$$
\begin{cases}\frac{\partial E(x, t)}{\partial t}-\frac{a D}{q} \Delta v(x, t) \leqslant \frac{k\left(\frac{\lambda}{d}+\varepsilon_{1}\right)}{1+\alpha\left(\frac{\lambda}{d}+\varepsilon_{1}\right)} v(x, t)-\frac{a m}{q} v(x, t)-\frac{b p}{c} z(x, t), & \text { in } \Omega \times\left[T_{1},+\infty\right), \\ \frac{\partial E(x, t)}{\partial v}=0, & \text { on } \partial \Omega \times\left[T_{1},+\infty\right), \\ E(x, t)=w(x, t)+\frac{a}{q} v(x, t)+\frac{p}{c} z(x, t), & \text { in } \Omega .\end{cases}
$$

The comparison principle and assumption $R_{0}<1$ show that

$$
\limsup _{t \rightarrow+\infty} E(x, t) \leqslant 0 \text {. }
$$

Combining with the positivity of solutions, we obtain that

$$
\lim _{\mathrm{t} \rightarrow+\infty} w(x, t)=\lim _{\mathrm{t} \rightarrow+\infty} v(x, t)=\lim _{\mathrm{t} \rightarrow+\infty} z(x, t)=0 \quad \text { in } \bar{\Omega} .
$$

Moreover, there exists $T_{3}>T_{1}$ such that $v(x, t) \leqslant \varepsilon_{3}$ in $\Omega \times\left[T_{3},+\infty\right)$ for an arbitrary constant $\varepsilon_{3}>0$. From 
the first equation of (1.4), we have

$$
\frac{\partial u(x, t)}{\partial t} \geqslant \lambda-d u(x, t)-\frac{k}{\alpha} \varepsilon_{3} \quad \text { for } \quad(x, t) \in \Omega \times\left[T_{3},+\infty\right),
$$

which leads to

$$
\liminf _{t \rightarrow+\infty} u(x, t) \geqslant \frac{\lambda-\frac{k}{\alpha} \varepsilon_{3}}{d}
$$

and

$$
\liminf _{t \rightarrow+\infty} u(x, t) \geqslant \frac{\lambda}{d}
$$

by the continuity as $\varepsilon_{3} \rightarrow 0$. Then we have $\lim _{t \rightarrow+\infty} \mathfrak{u}(x, t)=\frac{\lambda}{d}$. This proves the theorem.

Remark 3.2. From Theorem 3.1, we know that $E_{0}=(\lambda / d, 0,0,0)$ attracts every solution of system (1.4) when the basic reproduction number is smaller than one. This means that virus population vanishes and the disease will go away.

\subsection{Global stability of the immune-free equilibrium}

To discuss the global stability of immune-free equilibrium $E_{1}$, we need the following lemma from [7].

Lemma 3.3. For the following system

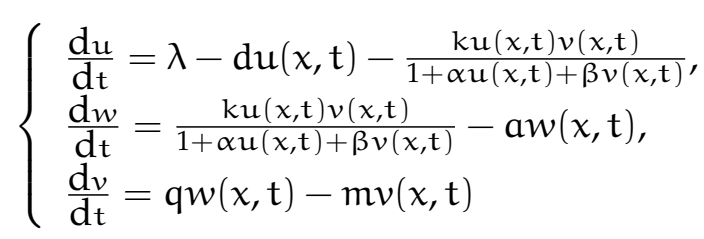

with initial value conditions

$$
u(0)=\max _{x \in \bar{\Omega}}\{u(x, 0)\}, w(0)=\max _{x \in \bar{\Omega}}\{w(x, 0)\}, v(0)=\max _{x \in \bar{\Omega}}\{v(x, 0)\},
$$

the unique positive equilibrium $\hat{\mathrm{E}}_{1}=\left(\mathrm{u}_{1}, w_{1}, v_{1}\right)$ is globally asymptotically stable when $\mathrm{R}_{0}>1$, where $\mathrm{u}_{1}, w_{1}, v_{1}$, and $\mathrm{R}_{0}$ are defined in Lemma 1.1.

Theorem 3.4. If $\mathrm{R}^{0}<1<\mathrm{R}_{0}$, then the immune-free equilibrium $\mathrm{E}_{1}$ of system (1.4) is globally asymptotically stable.

Proof. From the first three equations of system (1.4), we have

$$
\begin{cases}\frac{\partial u(x, t)}{\partial t}=\lambda-d u(x, t)-\frac{k u(x, t) v(x, t)}{1+\alpha u(x, t)+\beta v(x, t)}, & \\ \frac{\partial w(x, t)}{\partial t} \leqslant \frac{k u(x, t) v(x, t)}{1+\alpha u(x, t)+\beta v(x, t)}-a w(x, t), & \\ \frac{\partial v(x, t)}{\partial t}-D \Delta V(x, t)=q w(x, t)-m v(x, t), & \text { in } \Omega \times[0,+\infty), \\ \frac{\partial u(x, t)}{\partial v}=\frac{\partial w(x, t)}{\partial v}=\frac{\partial v(x, t)}{\partial v}=0, & \text { on } \partial \Omega \times[0,+\infty), \\ u(x, 0) \geqslant 0, w(x, 0) \geqslant 0, v(x, 0) \geqslant 0, & \text { in } \Omega .\end{cases}
$$

By the comparison system (3.1), we can obtain

$$
\lim _{t \rightarrow+\infty} \sup u(x, t) \leqslant u_{1}, \lim _{t \rightarrow+\infty} \sup w(x, t) \leqslant w_{1}, \lim _{t \rightarrow+\infty} \sup v(x, t) \leqslant v_{1} .
$$

As $\mathrm{R}^{0}<1$, it can be deduced that $w_{1}<\mathrm{b} / \mathrm{c}$. We may choose appropriate $\varepsilon_{4}>0$ such that $\mathrm{c}\left(\boldsymbol{w}_{1}+\varepsilon_{4}\right)-$ $\mathrm{b} \leqslant 0$. For this $\varepsilon_{4}$, there exists $\mathrm{T}_{4}>0$ such that $w(x, t) \leqslant w_{1}+\varepsilon_{4}$ for $(x, t) \in \Omega \times\left[T_{4},+\infty\right)$. 
From the fourth equation of system (1.4), we get

$$
\frac{\partial z(x, t)}{\partial t} \leqslant\left(c\left(w_{1}+\varepsilon_{4}\right)-b\right) z(x, t) \leqslant 0 .
$$

Incorporating into the positivity of solutions, we can obtain that $\lim _{t \rightarrow+\infty} z(x, t)=0$. Then, choose sufficiently small $\varepsilon_{5}>0$ and $T_{5}>0$ such that $\hat{R}^{0}<1<\hat{R}_{0}$ and $z(x, t) \leqslant \varepsilon_{5} / p$ for $(x, t) \in \Omega \times\left[T_{5},+\infty\right)$, where

$$
\hat{R}^{0}=\frac{\lambda k q c+\left(a+\varepsilon_{5}\right)^{2} b m \alpha}{\left(a+\varepsilon_{5}\right)(c d m+b d q \beta+b k q+c m \alpha \lambda)}, \quad \hat{R}_{0}=\frac{\lambda k q}{m\left(a+\varepsilon_{5}\right)(d+\alpha \lambda)} .
$$

Thus,

$$
\begin{cases}\frac{\partial u(x, t)}{\partial t}=\lambda-d u(x, t)-\frac{k u(x, t) v(x, t)}{1+\alpha u(x, t)+\beta v(x, t)}, & \\ \frac{\partial w(x, t)}{\partial t} \geqslant \frac{k u(x, t) v(x, t)}{1+\alpha u(x, t)+\beta v(x, t)}-\left(a+\varepsilon_{5}\right) w(x, t), & \\ \frac{\partial v(x, t)}{\partial t}-D \Delta V(x, t)=q w(x, t)-m v(x, t), & \text { in } \Omega \times\left[T_{5},+\infty\right), \\ \frac{\partial u(x, t)}{\partial v}=\frac{\partial w(x, t)}{\partial v}=\frac{\partial v(x, t)}{\partial v}=0, & \text { on } \partial \Omega \times\left[T_{5},+\infty\right), \\ u(x, 0) \geqslant 0, w(x, 0) \geqslant 0, v(x, 0) \geqslant 0, & \text { in } \Omega .\end{cases}
$$

Reconsidering the comparison system (3.1) and substituting $\left(a+\varepsilon_{5}\right)$ for $a$, we have

$$
\limsup _{t \rightarrow+\infty} u(x, t) \geqslant \hat{u}_{1}, \limsup _{t \rightarrow+\infty} w(x, t) \geqslant \hat{w}_{1}, \limsup _{t \rightarrow+\infty} v(x, t) \geqslant \hat{v}_{1}
$$

where

$$
\hat{u}_{1}=\frac{\left(a+\varepsilon_{5}\right) m+\beta \lambda q}{k q+\beta d q-\left(a+\varepsilon_{5}\right) m \alpha}, \quad \hat{w}_{1}=\frac{\lambda k q\left(\hat{R}_{0}-1\right)}{\left(a+\varepsilon_{5}\right) \hat{R}_{0}\left(k q+\beta d q-\left(a+\varepsilon_{5}\right) m \alpha\right)}, \quad \hat{v}_{1}=\frac{q}{m} \hat{w}_{1} .
$$

As $\varepsilon_{5} \rightarrow 0$, we have

$$
\lim _{t \rightarrow+\infty} u(x, t) \geqslant u_{1}, \lim _{t \rightarrow+\infty} w(x, t) \geqslant w_{1}, \lim _{t \rightarrow+\infty} v(x, t) \geqslant v_{1} .
$$

Therefore the immune-free equilibrium $E_{1}$ is globally asymptotically stable. The proof is complete.

Remark 3.5. From Theorem 3.1 and Theorem 3.4, it can be shown that system (1.4) is not persistent when the immune response reproduction number $\mathrm{R}^{0}$ is smaller than one.

\subsection{Global stability of the interior equilibrium}

Theorem 3.6. If $\mathrm{R}^{0}>1$, then the interior equilibrium $\mathrm{E}^{*}$ of system (1.4) is globally asymptotically stable.

Proof. Let $(u(x, t), w(x, t), v(x, t), z(x, t))$ be the solution of (1.4). We define the following Lyapunov function

$$
\begin{aligned}
\mathrm{V}(\mathrm{t})= & \int_{\Omega}\left[\frac{1+\beta v^{*}}{1+\alpha \mathrm{u}^{*}+\beta v^{*}}\left(\mathrm{u}-\mathrm{u}^{*}-\mathrm{u}^{*} \ln \frac{\mathrm{u}}{\mathrm{u}^{*}}\right)+\left(w-w^{*}-w^{*} \ln \frac{w}{w^{*}}\right)\right. \\
& \left.+\frac{\mathrm{a}+\mathrm{p} z^{*}}{\mathrm{q}}\left(v-v^{*}-v^{*} \ln \frac{v}{v^{*}}\right)+\frac{\mathrm{p}}{\mathrm{c}}\left(z-z^{*}-z^{*} \ln \frac{z}{z^{*}}\right)\right] \mathrm{d} x .
\end{aligned}
$$

By direct computations, it follows that

$$
\begin{aligned}
\frac{\mathrm{dV}(\mathrm{t})}{\mathrm{dt}} & =\int_{\Omega}\left[\frac{1+\beta v^{*}}{1+\alpha \mathrm{u}^{*}+\beta v^{*}} \frac{\mathrm{u}-\mathrm{u}^{*}}{\mathrm{u}} \frac{\partial \mathrm{u}}{\partial \mathrm{t}}+\frac{w-w^{*}}{w} \frac{\partial w}{\partial \mathrm{t}}+\frac{\mathrm{a}+\mathrm{p} z^{*}}{\mathrm{q}} \frac{v-v^{*}}{v} \frac{\partial v}{\partial t}+\frac{p}{\mathrm{c}} \frac{z-z^{*}}{z} \frac{\partial z}{\partial \mathrm{t}}\right] \mathrm{d} x \\
& =\frac{\mathrm{D}\left(\mathrm{a}+\mathrm{p} z^{*}\right)}{\mathrm{q}} \int_{\Omega} \frac{v-v^{*}}{v} \Delta v \mathrm{~d} x+\int_{\Omega}\left[\left(1-\mathrm{a} w^{*}-p w^{*} z^{*}\right)\left(\lambda-\mathrm{du}-\frac{\mathrm{kuv}}{1+\alpha u+\beta v}\right)\right.
\end{aligned}
$$




$$
\begin{aligned}
& +\left(1-\frac{w^{*}}{w}\right)\left(\frac{k u v}{1+\alpha u+\beta v}-a w-p w z\right) \\
& \left.+\frac{a+p z^{*}}{q}\left(1-\frac{v^{*}}{v}\right)(q w-m v)+\frac{p}{c}\left(1-\frac{z^{*}}{z}\right)(c w z-b z)\right] d x \\
& \triangleq I_{1}+I_{2} .
\end{aligned}
$$

From Green's formula and homogeneous Neumann boundary conditions, we have

$$
\mathrm{I}_{1}=-\frac{\mathrm{D}\left(\mathrm{a}+\mathrm{p} z^{*}\right)}{\mathrm{q}} \int_{\Omega} \frac{v^{*}|\nabla v|^{2}}{v^{2}} \mathrm{~d} x \leqslant 0
$$

Note that

$$
\lambda=d u^{*}+a w^{*}+p w^{*} z^{*}, \quad k u^{*} v^{*}=\left(a w^{*}+p w^{*} z^{*}\right)\left(1+\alpha u^{*}+\beta v^{*}\right), \quad \frac{m}{q}=\frac{w^{*}}{v^{*}},
$$

we can obtain

$$
\begin{aligned}
& \mathrm{I}_{2}=\int_{\Omega}\left[\mathrm{du} u^{*}\left(1-\frac{\mathrm{u}}{\mathrm{u}^{*}}-\frac{\mathrm{u}^{*}}{\mathrm{u}} \frac{1+\alpha \mathrm{u}+\beta v^{*}}{1+\alpha \mathrm{u}^{*}+\beta v^{*}}+\frac{1+\alpha u+\beta v^{*}}{1+\alpha \mathrm{u}^{*}+\beta v^{*}}\right)\right. \\
& +\left(a w^{*}+p w^{*} z^{*}\right)\left(1-\frac{u^{*}}{u} \frac{1+\alpha u+\beta v^{*}}{1+\alpha u^{*}+\beta v^{*}}+\frac{v}{v^{*}} \frac{1+\alpha u+\beta v^{*}}{1+\alpha u+\beta v}\right) \\
& \left.+\left(a w^{*}+p w^{*} z^{*}\right)\left(1-\frac{w^{*} u v}{w u^{*} v^{*}} \frac{1+\alpha u^{*}+\beta v^{*}}{1+\alpha u+\beta v}\right)+\left(a w^{*}+p w^{*} z^{*}\right)\left(1-\frac{v}{v^{*}}-\frac{w v^{*}}{w^{*} v}\right)\right] d x \\
& =\int_{\Omega}\left[\left(a w^{*}+p w^{*} z^{*}\right)\left(-1-\frac{v}{v^{*}}+\frac{v}{v^{*}} \frac{1+\alpha u+\beta v^{*}}{1+\alpha u+\beta v}+\frac{1+\alpha u+\beta v}{1+\alpha u+\beta v^{*}}\right)\right. \\
& -\frac{d\left(1+\beta^{*}\right)}{u\left(1+\alpha u^{*}+\beta v^{*}\right)}\left(u-u^{*}\right)^{2}+\left(a w^{*}+p w^{*} z^{*}\right)\left(4-\frac{u^{*}}{u} \frac{1+\alpha u+\beta v^{*}}{1+\alpha u^{*}+\beta v^{*}}\right. \\
& \left.\left.-\frac{w^{*} u v}{w u^{*} v^{*}} \frac{1+\alpha u^{*}+\beta v^{*}}{1+\alpha u+\beta v}-\frac{w v^{*}}{w^{*} v}-\frac{1+\alpha u+\beta v}{1+\alpha u+\beta v^{*}}\right)\right] \mathrm{d} x \\
& =\int_{\Omega}\left[-\frac{\mathrm{d}\left(1+\beta v^{*}\right)}{\mathfrak{u}\left(1+\alpha u^{*}+\beta v^{*}\right)}\left(u-u^{*}\right)^{2}-\left(a w^{*}+p w^{*} z^{*}\right) \frac{\beta(1+\alpha u)\left(v-v^{*}\right)^{2}}{v^{*}(1+\alpha u+\beta v)\left(1+\alpha u^{*}+\beta v^{*}\right)}\right. \\
& \left.+w^{*}\left(a+p z^{*}\right)\left(4-\frac{u^{*}\left(1+\alpha u+\beta v^{*}\right)}{u\left(1+\alpha u^{*}+\beta v^{*}\right)}-\frac{w^{*} u v\left(1+\alpha u^{*}+\beta v^{*}\right)}{w u^{*} v^{*}(1+\alpha u+\beta v)}-\frac{w v^{*}}{w^{*} v}-\frac{1+\alpha u+\beta v}{1+\alpha u+\beta v^{*}}\right)\right] \mathrm{d} x .
\end{aligned}
$$

Since the arithmetic mean is greater than or equal to the geometric mean, it is clear that $I_{2} \leqslant 0$ and the equality holds if and only if $u=u^{*}, w=w^{*}, v=v^{*}$, and $z=z^{*}$.

Therefore, the interior equilibrium $E^{*}$ is globally asymptotically stable when $R^{0}>1$ by the LaSalle invariance principle [3]. The theorem is proved.

\section{Conclusions}

In this paper, we have considered the well-posedness of solutions and global stability of a viral model with CTL immune response, which is a reaction-diffusion system with Beddington-DeAngelis incidence rate. In truth, the delayed model without diffusion is presented and the existence of Hopf bifurcation is investigated in [24]. Besides, the model in the present paper is more generalized than those in [7, 11, 18, 19].

According to those theorems on global stability in previous section, we can find that the global stability of three nonnegative equilibria in system (1.4) is entirely unrelated to diffusion. In other words, spatial diffusion has no influence on the global stability of equilibria. 
From the previous analyses, we can also obtain the permanence of system. Specifically, the viral system is not persistent when the immune response reproduction number $R^{0}$ is smaller than one and is permanent when $\mathrm{R}^{0}$ is greater than one.

Also, it has been found that time delay in reaction-diffusion epidemic model may induce some complex dynamics, such as bifurcation phenomenon [10], Turing pattern [16], and so on. In the future, we will continue investigating the combined influence of time delay and spatial diffusion on the dynamics of virus dynamics model with CTL immune response.

\section{Acknowledgment}

This work is supported by the Key Project for Excellent Young Talents Fund Program of Higher Education Institutions of Anhui Province (gxyqZD2016100) and the NSF of Education Bureau of Anhui Province (KJ2017A432).

\section{References}

[1] N. C. Chí, E. ÁvilaVales, G. García Almeida, Analysis of a HBV model with diffusion and time delay, J. Appl. Math., 2012 (2012), 25 pages. 1

[2] S. A. Gourley, J. W.-H. So, Dynamics of a food-limited population model incorporating nonlocal delays on a finite domain, J. Math. Biol., 44 (2002), 49-78. 1

[3] J. K. Hale, S. M. Verduyn Lunel, Introduction to functional-differential equations, Applied Mathematical Sciences, Springer-Verlag, New York, (1993). 3.3

[4] K. Hattaf, N. Yousfi, A. Tridane, Stability analysis of a virus dynamics model with general incidence rate and two delays, Appl. Math. Comput., 221 (2013), 514-521. 1

[5] D. Henry, Geometric theory of semilinear parabolic equations, Lecture Notes in Mathematics, Springer-Verlag, BerlinNew York, (1993). 2

[6] A. V. Herz, S. Bonhoeffer, R. M. Anderson, R. M. May, M. A. Nowak, Viral dynamics in vivo: limitations on estimates of intracellular delay and virus decay, Proc. Natl. Acad. Sci. U.S.A., 93 (1996), 7247-7251. 1

[7] G. Huang, W.-B. Ma, Y. Takeuchi, Global properties for virus dynamics model with Beddington-DeAngelis functional response, Appl. Math. Lett., 22 (2009), 1690-1693. 1, 1, 3.2, 4

[8] A. Korobeinikov, Global properties of basic virus dynamics models, Bull. Math. Biol., 44 (2004), 879-883. 1

[9] D. Li, W.-B. Ma, Asymptotic properties of a HIV-1 infection model with time delay, J. Math. Anal. Appl., 335 (2007), 683-691. 1

[10] P.-P. Liu, Periodic solutions in an epidemic model with diffusion and delay, Appl. Math. Comput., 265 (2015), $275-291$. 4

[11] M. A. Nowak, C. R. M. Bangham, Population dynamics of immune responses to persistent viruses, Science, 272 (1996), 74-79. 1, 1, 4

[12] M. A. Nowak, S. Bonhoeffer, A. M. Hill, R. Boehme, H. C. Thomas, H. McDade, Viral dynamics in hepatitis B virus infection, Proc. Natl. Acad. Sci. U.S.A., 93 (1996), 4398-4402.

[13] M. A. Nowak, R. M. May, Virus dynamics: mathematical principles of immunology and virology, Oxford University Press, Oxford, (2000). 1

[14] L.-B. Rong, Alan S. Perelson, Modeling HIV persistence, the latent reservoir, and viral blips, J. Theoret. Biol., 260 (2009), 308-331. 1

[15] X.-Y. Song, A. U. Neumann, Global stability and periodic solution of the viral dynamics, J. Math. Anal. Appl., 329 (2007), 281-297. 1

[16] Y. Wang, J.-D. Cao, G.-Q. Sun, J. Li, Effect of time delay on pattern dynamics in a spatial epidemic model, Phys. A, 412 (2014), 137-148. 4

[17] K.-F. Wang, A.-J. Fan, A. Torres, Global properties of an improved hepatitis B virus model, Nonlinear Anal. Real World Appl., 11 (2010), 3131-3138. 1

[18] X. Wang, Y.-D. Tao, X.-Y. Song, Global stability of a virus dynamics model with Beddington-DeAngelis incidence rate and CTL immune response, Nonlinear Dynam., 66 (2011), 825-830. 1, 1, 1, 4

[19] K.-F. Wang, W.-D. Wang, Propagation of HBV with spatial dependence, Math. Biosci., 210 (2007), 78-95. 1, 1, 4

[20] H.-B. Wang, R. Xu, Z.-W. Wang, H. Chen, Global dynamics of a class of HIV-1 infection models with latently infected cells, Nonlinear Anal. Model. Control, 20 (2015), 21-37. 1

[21] J.-H. Wu, Theory and applications of partial functional-differential equations, Applied Mathematical Sciences, SpringerVerlag, New York, (1996). 2

[22] Y.-N. Xiao, H.-Y. Miao, S.-Y. Tang, H.-L. Wu, Modeling antiretroviral drug responses for HIV-1 infected patients using differential equation models, Adv. Drug Deliv. Rev., 65 (2013), 940-953. 1 
[23] J.-H. Xu, Y. Geng, J.-Y. Hou, Global dynamics of a diffusive and delayed viral infection model with cellular infection and nonlinear infection rate, Comput. Math. Appl., 73 (2017), 640-652. 1

[24] Y. Yang, Stability and Hopf bifurcation of a delayed virus infection model with Beddington-DeAngelis infection function and cytotoxic T-lymphocyte immune response, Math. Methods Appl. Sci., 38 (2015), 5253-5263. 4

[25] Y. Yang, Y.-C. Xu, Global stability of a diffusive and delayed virus dynamics model with Beddington-DeAngelis incidence function and CTL immune response, Comput. Math. Appl., 71 (2016), 922-930. 1

[26] Y.-Y. Zhang, Z.-T. Xu, Dynamics of a diffusive HBV model with delayed Beddington-DeAngelis response, Nonlinear Anal. Real World Appl., 15 (2014), 118-139. 1 